\title{
La escuela no es una empresa, ni la educación un negocio ${ }^{1}$
}

Deyby Rodrigo Espinosa Gómez²

Universidad de Antioquia, Colombia

deibit05@hotmail.com

1 Artículo de reflexión.

2 Licenciado en Ciencias Sociales. Universidad de Antioquia. Colegio Parroquial de San Francisco de Asís. 


\title{
La escuela no es una empresa, ni la educación un negocio
}

\section{Resumen}

El presente artículo propone una reflexión crítica sobre la influencia del discurso empresarial en la escuela, el cual considera a la educación como un bien esencialmente privado y cuyo valor es ante todo económico. Hoy no cesa de escucharse cómo conceptos propios del mundo empresarial y mercantil se adhieren a la educación: "calidad», "competencias», «indicadores», "excelencia», "estándares», "evaluación por resultados», "pruebas estandarizadas», "oferta y demanda», "cliente y servicio», etc. Esto ha hecho que se sobrevalore esta clase de conceptos y no a la educación misma; es decir, los conceptos empresariales se usan como expresión genérica y como única verdad para caracterizar la educación.

En ese sentido, en primer lugar, se expondrá las trampas, efectos y consecuencias de uno de los conceptos originarios del mundo empresarial como lo es el de calidad de la educación. En segundo lugar, surge la necesidad y la urgencia de develar prácticas alternas en defensa de una escuela que recupere la educación como valor social.

Palabras clave: calidad, calidad de la educación, escuela, educación, maestro.

\section{The School is not a Company, nor Education a Business}

\begin{abstract}
This article proposes a critical reflection on the influence of business discourse in the school, which considers education as an essentially private good and whose value is above all economic. Today, concepts such as "quality", "competences", "indicators", "excellence", "standards", "performance evaluation", "standardized tests" 'Supply and demand', 'customer and service', etc., which belong to business, are attached to education. This has made this type of concepts to be overestimated and not education itself; that is, business concepts are used as a generic expression and as the only truth to characterize education.

In this sense, first, the traps, effects and consequences of one of the original concepts of the business world will be exposed, as is the quality of education. Secondly, there arises the need and urgency to unveil alternative practices in defense of the school, as institution, that recovers education as a social value.
\end{abstract}

Key words: Quality, Quality of Education, School, Education, Teacher.

\section{A escola não é uma empresa, nem a educação um negócio}

\section{Resumo}

Este artigo propõe uma reflexão crítica sobre a influência do discurso corporativo na escola, que considera a educação como um bem essencialmente privado e cujo valor é principalmente económico. Hoje não cessa de se escutar como conceitos próprios do mundo empresarial e mercantil se aderem à educação: "qualidade», "concorrências», «indicadores», "excelência», "regulares», "avaliação por resultados», "provas padronizadas», "oferta e demanda», "cliente e serviço», etc. Isto tem feito que se exagere esta classe de conceitos e não à educação mesma; isto é, os conceitos empresariais usam-se como expressão genérica e como única verdade para caracterizar a educação.

Nesse sentido, em primeiro lugar, se exporá as armadilhas, efeitos e consequências de um dos conceitos originarios do mundo empresarial como o é o de qualidade da educação. Em segundo lugar, surge a necessidade e a urgência de develar práticas alternadas em defesa duma escola que recupere a educação como valor social.

Palavras-chave: qualidade, qualidade da educação, escola, educação, maestro. 


\section{Introducción}

Hace ciento treinta años, después de visitar el país de las maravillas, Alicia se metió en un espejo para descubrir el mundo al revés. Si Alicia renaciera en nuestros días, no necesitaría atravesar ningún espejo: le bastaría con asomarse a la ventana.

Al fin del milenio, el mundo al revés está a la vista: es el mundo tal cual es, con la izquierda a la derecha, el ombligo en la espalda y la cabeza en los pies.

Galeano (1998)

Esa frase del pensador uruguayo Eduardo Galeano (1998) es útil para comenzar este artículo, dado que la escuela de hoy parece estar patas arriba, ya no está sostenida por la «cultura pedagógica», sino más bien por la «cultura del mercado». El nuevo orden mundial, de la mano de las organizaciones internacionales — OMEC, OCDE, Banco Mundial, FMl, y la Comisión Europea-, contribuye a que la escuela pierda progresivamente su autonomía para someterse a políticas educativas que respondan a las necesidades del mercado. El modelo escolar y educativo que tiende a imponerse está fundado, en primer lugar, en el sometimiento más directo de la escuela a los objetivos de competitividad que prevalecen en la economía globalizada (Laval, 2004).

A propósito de lo anterior, la profesora Cecilia Salazar (2012) refiere que la administración educativa actual parece empeñada en diseñar planes para las escuelas en los que se trata a los estudiantes como productos, a los padres como clientes y a las escuelas como empresas, se trata cada vez más de convertir a la escuela en una institución «económicamente útil». Avizorando este panorama, Martin Heidegger (1981) dijo de manera contundente en Carta sobre el humanismo: «La educación está en lo seco». Para Guillermo Hoyos (2009), Heidegger no se equivocó en su señalamiento, pues la educación se ha positivizado, se ha distraído en otros temas y se ha olvidado de la cosa misma, la condición humana.

La condición humana parece desaparecer, y consigo, el derecho a la diferencia, para dar paso a una educación que se entiende como un producto y no como un proceso. El sistema educativo, de la mano de la empresa, pretende educar a las personas en equidad mientras prioriza la competitividad, habla de diversidad y establece unos parámetros únicos de calidad educativa para todos, pretende que los estudiantes sean creativos y en las pruebas estandarizadas estos deben responder de memoria sin importar sus necesidades. Ante esta situación, el sociólogo Zygmunt Bauman (2012) consideró que la crisis educativa reside en que se enseña a ser mejor que el otro, que hay que competir constantemente; al mismo tiempo, la educación persigue la naturalización y normalización de los diversos modos de pensar, instaurándose como principio que controla e influye el comportamiento humano. 
La escuela parece estar más preocupada por competir y seleccionar a los mejores que en comprender la coexistencia y circulación de los diferentes ritmos de aprendizaje que habitan en ella. El profesor Alfredo Ghiso (2013) precisa que nuestro sistema dejó de pensar lo educativo e instaló una lógica racional tecnoburocrática incapaz e inhabilitada para leer lo humano más allá de las leyes de la productividad, los daños colaterales, la oferta, la demanda y la competitividad. De esta forma, estamos ante un sistema educativo en el cual prima el resultado y la competencia, mas no la dimensión ética; lo humano se reemplaza por un número en un papel, interesa que quienes ganen sean los mejores, los más calificados, a la vez que se identifica y descarta a los "peores". En relación con la competitividad en la escuela, el defensor de la educación progresiva Alfie Kohn (2014) sostiene que la competitividad atenta contra el aprendizaje, destruye las relaciones humanas, ya que su idea central es que los demás son potenciales obstáculos para el éxito propio; asimismo, crea desprecio hacia los otros, promueve la agresividad, estimula la trampa, el engaño, la estafa y evita el surgimiento de conductas cooperativas.

Siguiendo a Kohn, lo que parece motivar a los colegios es alcanzar puntajes, esto hace que los niños odien la institución y duden de sus propias capacidades; además, les enseña que conseguir buenas notas significa ser mejor que el resto.

A continuación, se expone un concepto originario del mundo empresarial que por su naturaleza y característica demanda una constante competencia y comparación entre las personas e instituciones, un concepto que favorece que la escuela cada vez más se convierta en una empresa; hablamos de la calidad de la educación.

\section{La calidad de la educación: un concepto no pensado por la educación}

La eficacia y la competitividad se convierten en señuelos que condicionan las concepciones, las actitudes y las prácticas de las personas inmersas en la cultura hegemónica. La eficacia, como sinónimo de la calidad, es el mito contemporáneo.

Le Mouël (1992)

La calidad no es un concepto gestado y pensado por la educación, es un concepto originario del discurso empresarial. En un principio, calidad se utilizaba para referirse a un producto material, por ejemplo, un libro, una mesa o una silla, para indicar, por decir algo, que las hojas de un libro son de buena calidad. Esa denominación se usaba para catalogar objetos materiales, pero desde la década de 1980 el concepto se adhiere, de la mano del neoliberalismo, a cualquier servicio público para permitir la comparación, adherencia de la que no se salvó ni la educación, ni el propio ser humano. 
Es bueno recordar, como lo señala el historiador y educador Renán Vega (2012), que el concepto de calidad educativa se usó por primera vez en el año de 1966 con el economista Charles Beevy en su libro La calidad de la educación en los países en desarrollo. Posteriormente, en el año de 1968 se retoma con el experto de la Unesco Philips Cooms en su libro La crisis mundial de la educación. Pero solamente hasta 1983 en los Estados Unidos, con el Informe de la Comisión Nacional de Excelencia en Educación, se habla directamente de «calidad educativa» como un lineamiento de política por parte de un país. Merced a esta comisión se propuso evaluar la calidad de los procesos educativos en la escuela y la universidad, también, comparar la educación e instituciones educativas a nivel internacional.

En la década de 1980, el concepto comienza a ocupar un lugar destacado en las agendas de la política pública en educación y, de manera expresa, en el contexto latinoamericano (Vidal, 2007), convirtiéndose en uno de los puntos sobre los cuales han girado las reformas educativas hasta la actualidad. La calidad se instituye en los distintos escenarios sociales y se instala en las agendas gubernamentales y de los organismos multilaterales mediante la creación de una compleja red de sinergias que impactan a la educación en sus dimensiones epistemológicas, políticas y pedagógicas. Así mismo, la calidad de la educación expresa, y es a su vez expresión de, los procesos de globalización y transnacionalización de la cultura (Orozco, Olaya \& Villate, 2009).

Ante la situación planteada, el profesor Casseaus (2007) coincide en que el giro en la política de educación a nivel mundial no tardó en Ilegar a América Latina. El giro consistió en que el centro de la política dejó de ser la educación y se dio paso a la calidad. Para ello, la nueva política se focalizó en cambiar la regulación del sistema apoyándose en la naciente disciplina de la gestión educativa. No obstante, nunca se debatió acerca de lo que era calidad y, por tanto, nunca hubo siquiera un intento de consenso respecto a ese concepto. En palabras de Casseaus (2007):

[...] la ausencia de contenido y la ausencia de consenso acerca de la calidad nos jugó una mala pasada. Por algún motivo aún no esclarecido se dio por sobreentendido que todo el mundo sabía de qué se trataba la calidad de la educación, de tal manera, que cuando se formularon las políticas educativas desde esa época, bastaba con que se dijera que eran «para mejorar la calidad de la educación» para que fueran aceptadas por las autoridades. Sin embargo, éstas nunca fueron políticas de educación sino que fueron políticas de gestión. Las políticas educativas se centraron en la gestión del sistema, y nunca en la educación. (p. 72).

En este mismo orden y dirección, el educador Alberto Martínez (2004) señala que la política educativa en Latinoamérica encontró su sentido en «el valor central del mercado educativo» (p. 11), razón por la cual los principios de calidad, eficiencia y eficacia son considerados por los discursos dominantes como ejes centrales en sus propósitos y contenidos. De ahí que estas políticas educativas, con la participación 
e influencia del FMl y el BM, resaltan la construcción de una nueva racionalidad educativa basada en los logros de la calidad y la gestión educativa para hacer frente a un mundo más competitivo y globalizado.

Resulta oportuno profundizar sobre el concepto de calidad y su origen lexical. De acuerdo con La Real Academia Española (2014), calidad se define como: 1) propiedad o conjunto de propiedades inherentes a algo, que permite juzgar su valor; 2) buena calidad, superioridad o excelencia; 3) adecuación de un producto o servicio a las características especificadas.

En su trabajo sobre la calidad, y citando otros autores, Luengo y Casanova (2012) advierten que el concepto de calidad se redefine y se interesa por los buenos resultados, o sea, por todo aquello que tenga la capacidad de medirse $y$, en última instancia, de convertirse en un número, un dato, una gráfica..., para poder etiquetar, comparar o jerarquizar a los sujetos en un ejercicio de poder. Es allí donde este concepto es incompatible con la educación, pues la educación, las escuelas y la dignidad de las personas no pueden reducirse a la comparación reduccionista de la lupa utilitaria.

Para el sociólogo de la educación Rodrigo Jaramillo (2004), la calidad se fue configurando como expresión de «uso común» en los discursos académicos hasta convertirse en factor necesario para el aval de los mismos. De alguna manera, un amplio número de personas ha presumido que si un discurso que hace referencia a la educación no lleva explicito el término calidad, carece de valor.

Por otra parte, para Vega (2012), al introducirse la noción de calidad en la educación también se están involucrando aspectos coetáneos, propios del mundo empresarial, a los sistemas educativos, tales como control de calidad, mejoramiento de calidad, aseguramiento de la calidad. Con todas estas denominaciones tecnocráticas, a la educación se le atribuyen los mismos atributos que se le exigen a cualquier empresa: eficiencia, rendimiento, productividad incrementada a bajo costo, satisfacción de los clientes, competitividad, eficacia, innovación, rentabilidad, éxito y excelencia.

Cabe agregar, como lo señalan los profesores Jaramillo, Vélez y Espinosa (2011) en su trabajo Evaluación y calidad en la universidad: metáforas y perversiones, que el concepto de la calidad privilegia el imperio de los indicadores y el opacamiento de la educación, al tiempo que acentúa la prevalencia de ismos respecto a la certificación, el predominio de lo mercantil, la funcionalidad, los universalismos y el positivismo, promoviendo la exclusión y deshumanización. En otras palabras, la sobreestimación de la calidad ha propiciado el culto del gerencialismo y ha traído consigo que la calidad se anteponga a la educación y medie para que se imponga una visión deteriorada y desacreditada de lo público, haciendo presumir que, si no se está en la calidad, se está en lo opuesto. ¡Nada más atentatorio de la autonomía y los procesos formativos que la intención de generar un único estilo! (Jaramillo et al., 2011). 
Como la calidad no proviene del mundo de educativo, examinemos con más cuidado algunas de las trampas, efectos y consecuencias de este vocablo en la educación. Según Miguel Santos (1999) y Renán Vega (2012), puede encontrarse en la calidad las siguientes trampas intrínsecas y extrínsecas.

\section{Dimensiones intrínsecas}

\section{Simplificación abusiva}

Se presenta una simplificación consistente, el éxito se identifica con el rendimiento académico. La educación es minimizada al rendimiento y a la productividad. Con esta dimensión intrínseca se instauran ciertas evaluaciones para medir la calidad, evaluaciones en las que no se tienen en cuenta las labores intelectuales más ricas y complejas — como analizar, comprender, comparar...-, sino que se recurre a unos registros cuantitativos para medir habilidades, destrezas y competencias.

En este sentido, la evaluación de los centros educativos mide la calidad educativa en función de los resultados, y a partir de ahí se establece un ranking público, cuya finalidad es orientar a los padres para elegir a los mejores. En suma, los diferentes ritmos de aprendizaje se estigmatizan con la etiqueta «buena» o «mala», y los establecimientos educativos, por sus resultados en pruebas estandarizadas, como establecimientos de «calidad»y «mala calidad». Se simplifican las diferencias y los diversos ritmos de aprendizaje a un resultado, a la vez que se identifican a los exitosos y a los perdedores a través de una competencia, al mejor estilo del darwinismo, para construir un mercado educativo cada vez más competitivo.

\section{La confusión}

Se confunde la calidad con las condiciones materiales de la educación, es decir, que la primera está determinada por las instalaciones, espacios educativos o los medios didácticos más modernos, como si todo eso de manera automática generara una mejor educación. En definitiva, se entiende por educación de calidad aquella que asegura el éxito laboral del individuo, la calidad es incuestionable.

\section{Tecnificación}

Se centra en las evaluaciones de la calidad. Se da la impresión de que solamente cuando hay número y medida existe rigor y objetividad. Con las pruebas estandarizadas se clasifica y etiqueta a los estudiantes e instituciones de acuerdo a sus resultados; se condiciona las capacidades, y se predispone al fracaso o al éxito académico. Como señala Popham (1999), si los puntajes que obtiene una escuela en pruebas estandarizadas son altos, la gente piensa que el plantel educativo y su profesorado son eficaces, en cambio, si los puntajes en las pruebas estandarizadas son bajos, se considera que el plantel educativo y el cuerpo de profesores son 
ineficaces. Este proceso de medición, comparación y jerarquización entre sujetos, se convierte, como lo diría Calero y Choi (2012), en la «colonización de la educación» por medio de las evaluaciones de competencias y pruebas estandarizadas.

En tales pruebas no existen apreciaciones ni sociales ni éticas para juzgar a escuelas, estudiantes y profesores, sino puras y simples mediciones de rendimiento, como expresiones de una pretendida calidad educativa en las que no se consideran los contextos ni los condicionamientos sociales y, desde luego, no se tienen en cuenta las desigualdades sociales.

A efectos de estas pruebas, el defensor de la teoría de las inteligencias múltiples, Howard Gardner (1994), considera que existe un enorme deseo de convertir la educación en algo uniforme, de tratar a todos los estudiantes de la misma manera, sin tener en cuenta sus habilidades individuales, y aplicar el mismo tipo de evaluación unidimensional. Para Gardner, las pruebas estandarizadas son frecuentemente usadas para resaltar las debilidades de los estudiantes y no sus fortalezas, se convierten en evaluaciones de "talla única», sin considerar la diversidad del currículum y de los estudiantes. En resumen, con esta dimensión la diferencia se invisibiliza y homogeneiza, los estudiantes deben pensar igual, de la misma forma y en los mismos tiempos, el saber se reduce a un número en un papel.

\section{Dimensiones extrínsecas}

\section{La comparación arbitraria y abusiva}

Existe la comparación entre personas, experiencias y centros educativos (Santos, 1999) como si se pudiera juzgar con los mismos parámetros los diferentes ritmos de aprendizaje de los estudiantes, o como si se pudiera juzgar y comparar con los mismos criterios a un colegio de Colombia con uno de Finlandia, como si no existiesen contextos desiguales. En fin, se crea la cultura educativa de la rivalidad por el éxito, la lectura del propio contexto educativo desaparece para vivir el contexto educativo de otros países con mejores resultados.

\section{Perversión}

Se presenta la clasificación de los centros en buenos y malos (Santos, 1999). Con esta dimensión se lleva a presumir que lo privado es superior, de mejor calidad, que lo público, lo cual, más que una constatación, es un prejuicio que refuerza la lógica de la soberanía del consumidor, que decide qué es y qué no es de calidad. En suma, se pretende homogeneizar la educación mediante la imposición de estándares similares y subordinar las instituciones públicas a la lógica gerencial y mercantil que rige a las instituciones privadas. 
Como puede observarse, por medio de la adherencia de la calidad a la educación parece ocultarse problemas centrales y estructurales de la educación, cubriéndolos con la máscara de la eficiencia y productividad del sistema. De acuerdo con los razonamientos que se han venido realizando, cabe resaltar algunos rasgos generales de la calidad en la educación, como se ve en la tabla 1.

Tabla 1. Rasgos generales de la calidad en la educación

\begin{tabular}{|c|c|}
\hline COMPONENTE & CARACTERÍSTICAS \\
\hline Concepto de educación & $\begin{array}{l}\text { La educación se minimiza a merced de las estandarizaciones y la mer- } \\
\text { cantilización. Se entiende la educación como asunto de la productividad } \\
\text { articulada a la competitividad mundial. }\end{array}$ \\
\hline Paradigmas soportes & $\begin{array}{l}\text { Sistema neoliberal, que a través de la práctica de la teoría del capital hu- } \\
\text { mano busca el desarrollo del capital del conocimiento y las competencias } \\
\text { para el mercado. }\end{array}$ \\
\hline $\begin{array}{l}\text { Organismos internaciona- } \\
\text { les que la fomentan }\end{array}$ & OMEC, OCDE, BM — Banco Mundial—, FMI, y Comisión Europea. \\
\hline Función del Estado & $\begin{array}{l}\text { El Estado utiliza el concepto como muletilla para cualquier asunto con- } \\
\text { cerniente a la educación, como si su sólo nombre discursivo asegurase el } \\
\text { éxito. El Estado abusa del vocablo promoviendo su continuidad y conso- } \\
\text { lidación; simultáneamente, emplea la gestión educativa para competir a } \\
\text { nivel internacional. }\end{array}$ \\
\hline $\begin{array}{l}\text { Conceptos alusivos a la } \\
\text { calidad en educación }\end{array}$ & $\begin{array}{l}\text { Competencia, estándares, eficiencia, profesionalización, acreditación, } \\
\text { pruebas estandarizadas, calidad de la educación, desempeño, proyectos } \\
\text { educativos, gestión educativa, servicio, cliente, oferta y demanda. }\end{array}$ \\
\hline $\begin{array}{c}\text { Finalidad de la escuela } \\
\text { con la calidad }\end{array}$ & $\begin{array}{l}\text { Competir por ocupar los primeros puestos, hacer de la institución un es- } \\
\text { tablecimiento de calidad. } \\
\text { Formar para satisfacer las necesidades del mundo laboral. } \\
\text { Obtener altos resultados en pruebas estandarizadas. } \\
\text { Acreditación. }\end{array}$ \\
\hline
\end{tabular}

Fuente: elaboración propia.

\section{Pensar diferente}

Es evidente, entonces, que hay que desconfiar de aquellos discursos educativos que no aceptan la singularidad de las diferencias, sino que se esfuerzan por desdibujar y uniformar los diversos ritmos de aprendizajes. A veces, estos discursos no están diseñados para que se conviva con los otros, sino para que se compita con ellos. El énfasis de la educación debería ser la convivencia y la solidaridad antes que la rivalidad y la competencia, decía William Ospina (2008), y sólo podremos lograr ello a través de una educación diferente, en la cual se viva un contexto educativo sin competencias, pues el competir deteriora y estigmatiza las diferencias humanas. 
Humberto Maturana (2014) elevó su voz para recordarnos que la diferencia es legítima cuando coexiste en una cultura donde no hay que competir.

A lo largo de los planteamientos hechos, es evidente, pues, que no es lo mismo tener un modelo de calidad para las empresas, que tenerlo para nuestras escuelas. Antes de pensar un modelo de calidad para la escuela se debe, en primer lugar, comprender la escuela como un campo de humanidad, de humanidades y de humanización: de humanidad, porque es allí donde se aprende la defensa y promoción de los derechos humanos, donde adquieren valor las diferencias humanas; de humanidades, porque es en ella donde se promueve el aprendizaje, la crítica y reflexión frente a las realidades creadas por el hombre; de humanización, porque el compromiso de la escuela con la sociedad es contribuir a formar una sociedad más humana.

Tras el análisis del concepto de calidad en la educación es palmario el reconocimiento de algunas trampas terminológicas y consecuencias, las cuales alteran el estado de la educación de manera negativa y deshumanizante. El abuso discursivo y práctico de la noción de calidad en la educación ha propiciado el culto de las competencias y de la gestión en el mundo educativo. La calidad se sobreestima respecto a la educación y, de ese modo, se desarrolla la tendencia de atribuirle a esa noción de calidad la totalidad de la importancia, sea dentro de las acciones políticas o sociales.

En términos de calidad en la educación, lo más preocupante es su visión reduccionista y comparativa entre los seres humanos; además, fomenta el sofisma de que para aprender se debe competir, establece la cultura de la competencia como finalidad educativa, una rivalidad que atenta contra el derecho a ser diferente.

Mientras los sistemas educativos continúen pensando que la competencia es el camino, no se habrá comprendido el verdadero significado de la convivencia. Para ello no se necesita técnicos u operarios, sino seres sentipensantes, seres que actúen con el corazón usando la razón para construir una sociedad con los otros, no sobre otros. No hay, estrictamente hablando, un yo pienso, sino un nosotros pensamos. No es el yo pienso lo que constituye el nosotros pensamos, sino, por el contrario, es el nosotros pensamos lo que me permite pensar (Freire, 1990).

La transformación de estas realidades educativas no debe colocarse en los caminos de la utopía, ni tampoco pensar que hay un único futuro para recuperar la educación como valor social. Hoy, más que nunca, debe lucharse por una educación digna y no de calidad (Vega, 2012), para ello, se requiere cada vez más:

1. Un quehacer pedagógico al servicio de la problematización, la concientización y la humanización para el reconocimiento del contexto escolar. Es hora de vivir el contexto propio, y no el de otros países. 
2. Aulas en defensa de la dignidad humana, sobre la base de valores como el diálogo, la escucha, el respeto, la democracia y el reconocimiento a la diversidad.

3. Comprender que la educación es mucho más que una prueba estandarizada, ya que esta clase de pruebas coartan la diferencia, no pueden medir la creatividad, las destrezas de escritura, la resolución de problemas sociales o habilidades de pensamiento crítico. Con esta clase de pruebas los diferentes ritmos de aprendizajes se ajustan a una talla única sin considerar la diversidad de los estudiantes. Cabe agregar, como lo dijo Frederick Kelly, el creador de las pruebas estandarizadas: "Estos tests son demasiado poco desarrollados para aplicarlos y deben abandonarse» (Prince, 27 de septiembre de 2016).

4. Poner en práctica una pedagogía dialógica que enseñe a los estudiantes a pronunciar sus propias palabras, y no a repetir de memoria las palabras de un sistema mercantil, un sistema para la competencia entre hombres.

5. Políticas educativas que descontaminen a la educación de las manos de la empresa, políticas hacia una educación que no se limite a informar y adiestrar para la empresa y el trabajo, que no exagere el culto a la competitividad.

6. La escuela no es una competencia. La realidad de los nuevos tiempos exige de escuelas donde la diferencia no sea vista con los ojos de las competencias, sino con los ojos de la dignidad humana.

7. Es hora de una escuela que enseñe a los estudiantes a ser dueños de su palabra, capaces de criticar su realidad y su contexto sin estar mediatizados por una ideología dominante. Una escuela que posibilite la tarea de transformar el mundo.

La literatura ha sido más rica que muchas de las explicaciones actuales para caracterizar la educación y la escuela, y con claridad Eduardo Galeano (2000) nos recuerda que un hombre del pueblo de Neguá, en la costa colombiana, pudo subir al alto cielo, y que, al contemplar desde arriba la vida humana, dijo que somos un mar de fueguitos: «El mundo es eso, un montón de gente, un mar de fueguitos. Cada persona brilla con la luz propia entre todas las demás. No hay dos fuegos iguales» (2000, p. 5).

La escuela es eso, un mar de fueguitos, fuegos grandes y fuegos chicos, fuegos de todos los colores, fuegos con dignidad, fuegos con derechos, fuegos pensantes, fuegos nacidos para convivir y no para competir, fuegos con voces que aclaman que la escuela se ponga en pie, y no esté más patas arriba, fuegos que sueñan con que la escuela sea un espacio libre de condicionamientos y de competencias para el mundo económico. Es hora de soñar un mundo en el cual Alicia pueda atravesar al espejo, y encontrar una escuela en pie; en la cual, las competencias no sean el fin más importante, sino la dignidad humana; los estudiantes no sean clientes, 
ni los maestros obreros al mejor estilo de la empresa; sino que los maestros sean dignificados y respetados por los gobernantes y la sociedad. Un mundo en el cual se comprenda que la escuela no es una mercancía, ni la educación un negocio, porque la educación y el aprendizaje son derechos humanos. 


\section{Referencias}

Alberto, M. (2004). De la escuela expansiva a la escuela competitiva: dos modos de modernización en América Latina. Bogotá: Anthropos, Convenio Andrés Bello.

Bauman, Z. (20 de agosto de 2012). "Nos han dicho que hay que ser mejor que el otro, que hay que competir constantemente». Entrevista en Farodevigo.es. Decano de la Prensa Mundial. Recuperado de http://www.farodevigo.es/sociedad-cultura/2012/08/20/ bauman-han-dicho-hay-mejor/675624.html

Calero, J., y Choi, A. (2012). La evaluación como instrumento de política educativa. Presupuestos y Gasto Público, 67, 29-41.

Casassus, J. (2007). El precio de la evaluación estandarizada: la pérdida de calidad y la segmentación social. Revista Brasileira de Política e Administração da Educação / Periódico científico editado pela ANPAE, 23(1).

Freire, P. (1990). La importancia de leer y el proceso de liberación. México. Siglo XXI Editores.

Galeano, E. (1998). Patas arriba: la escuela del mundo al revés. Madrid: Siglo XXI.

Galeano, E. (2000). El libro de los abrazos. Siglo XXI.

Gardner, H. (1994). Estructuras de la mente: la teoría de las inteligencias múltiples. México: Fondo de Cultura Económica.

Ghiso, A. (2013). «De una lógica tecnoburocrática al encuentro educativo». En: El asombro de la educación: perspectivas, experiencias y propuestas para desarrollar nuevos paradigmas de aprendizaje. Santiago de Chile: Editorial Universidad Bolivariana S.A.

Heidegger, M. (1981). Carta sobre el humanismo. Buenos Aires: Ediciones del 80.

Hoyos, G. (2009). Educación para un nuevo humanismo. Magis, Revista Internacional de Investigación en Educación, 2, 425-433. Recuperado de http://revistas.javeriana.edu.co/ index.php/MAGIS/article/download/3395/2584

Jaramillo, R. (2004). La calidad de la educación: Un concepto de referencia. Revista Educación y Pedagogía, XVI(38). Recuperado de http://tesis.udea.edu.co/bitstream/10495/3078/1/ JaramilloRodrigo_2004_calidadeducacionconceptorefencia.pdf

Jaramillo, R., Vélez G., y Espinosa, D. (2011) Evaluación y Calidad en la universidad: Metáforas y perversiones. Universidad de Antioquia.

Kohn, A. (Julio, 2014). «Competir atenta contra el aprendizaje». El Tiempo. Recuperado de http://www.eltiempo.com/estilo-de-vida/educacion/entrevista-con-alfie-kohn-experto-eneducacion/14240918

Laval, C. (2004) La escuela no es una empresa. Barcelona: Paidós.

Le Mouel, J. (1992). Crítica de la eficacia. Ética, verdad y utopía de un mito contemporáneo. Barcelona: Paidós.

Lenguo, J., y Casanova, C. (2012). Mecanismos endógenos de privatización encubierta en la escuela pública. Políticas educativas de gestión de resultados y rendición de cuentas 
en Andalucía. Revista profesorado, 16(3), 111-126. Recuperado de http://www.ugr. es/ recfpro/rev163ART6.pdf

Maturana, H. (21 de mayo de 2014). «La educación es un espacio para que el niño se transforme en ciudadano ético». Entrevista en Radio Universidad de Chile. Recuperado de http://radio.uchile.cl/2014/05/21/humberto-maturana-la-educacion-es-un-espacio-paraque-el-nino-se-transforme-en-ciudadanos-etico/

Orozco, J., Olaya, A., y Villate, V. (2009) ¿Calidad de la educación o educación de calidad? una preocupación más allá del mercado". Revista iberoamericana de educación, (51), 161-181.

Ospina, W. (2008). La escuela de la noche. Bogotá: Editorial Norma.

Popham, W. (1999). ¿Por qué las pruebas estandarizadas no miden la calidad educativa? Biblioteca PREAL. Recuperado de file:///C:/Users/USER/Downloads/pruebas_estandarizadas_ no_miden_calidad_educativa_popham.pdf

Prince Ea, (27 de septiembre de 2016). Demandando al sistema educativo, [Archivo de video]. Recuperado de https://www.youtube.com/watch?v=G0Ece11tlvU

Real Academia Española. (2014). Diccionario de la lengua española (23a ed.). Recuperado de http://dle.rae.es/?id=6nVpk8P|6nXVL1Z

Salazar C. (2012). «Escuela o empresa: ¿Galton en las aulas?». Yo estudié en la pública. Ciudadan@s por la educación pública. Recuperado de https://www.yoestudieenlapublica. org/descargas/5articuloEscuelaEmpresa.pdf

Santos, M. (1999). Las trampas de la calidad. Revista Acción pedagógica, 8(2), 78-81. Recuperado de https://dialnet.unirioja.es/descarga/articulo/2973330.pdf.

Vega, R. (2012). La calidad educativa una noción neoliberal propia del darwinismo pedagógico. Rebelión. Recuperado de http://www.rebelion.org/noticia.php?id=142741

Vidal, L. (2007). Aproximación deconstructiva a la noción de calidad de la educación en el contexto latinoamericano. Revista Iberoamericana de Educación, 44(4). Recuperado de http://www.rieoei.org/ deloslectores/1959Vidal.pdf

Recibido: 6 de diciembre 2016

Aceptado: 24 de marzo 2017.

Cómo citar: Rodríguez, D. (2017). La escuela no es una empresa, ni la educación un negocio. Praxis Pedagógica, 21 (65-78). 